\title{
The aldosterone index could be used to diagnose the dominant gland in primary aldosteronism - a retrospective study
}

\author{
Sheng-Zhuo Liu', Liang Zhou', Tao Chen², Zhi-Hong Liu', Zheng-Ju Ren', Yu-Chun Zhu' \\ ${ }^{1}$ Department of Urology/Institute of Urology, West China Hospital, Sichuan University, Chengdu, Sichuan, China \\ ${ }^{2}$ Department of Endocrinology, West China Hospital, Sichuan University, Chengdu, Sichuan, China
}

\begin{abstract}
Introduction: Failed cannulation in the right adrenal vein, which makes the sampling results in the contralateral vein and inferior vena cava (IVC) nonsense, is the main obstacle of using adrenal vein sampling (AVS) in the lateralisation diagnosis in primary aldosteronism (PA). We performed a retrospective study to evaluate the specificity and sensitivity of using the aldosterone index (AI) in PA lateralisation diagnosis. Material and methods: We enrolled 116 patients who were diagnosed with PA and then underwent AVS in the West China Hospital of Sichuan University from April 2015 to April 2017. The AI, calculated by dividing the aldosterone concentration of the failed side by the aldosterone concentration of IVC, was used for lateralisation diagnosis if the cannulation was judged to be failed by traditional method. Patients with dominant adrenal gland based on successful AVS were included in subgroup $2(n=75)$, while the patients diagnosed with a dominant gland using AI method were enrolled in subgroup $1(n=41)$.

Results: No significant difference of clinical and biochemical findings between the two groups was detected ( $\mathrm{p}$ value after operation $>0.05)$. ROC analysis was performed to test the specificity and sensitivity based on the AI in subgroup 2 . The AUC for dominant gland detection was 0.76 , which resulted in $91.3 \%$ sensitivity and $67.53 \%$ specificity. The positive and negative likelihood ratios were 2.81 .

Conclusions: Our data suggested that the modified strategy using AI to diagnose the dominant gland in PA is an efficient method when cannulation has failed in the right side. (Endokrynol Pol 2020; 71 (1): 42-50)
\end{abstract}

Key words: primary aldosteronism; aldosterone-producing adenoma; adrenal vein sampling

\section{Introduction}

Primary aldosteronism (PA) is a major cause of secondary hypertension. According to epidemiological research, in about $5-15 \%$ of patients with hypertension it can be attributed to PA [1]. What is worse, about $65.6 \%$ of these patients suffer with secondary hypertension [2], and 15-20\% have treatment-resistant hypertension [3]. Primary aldosteronism is also characterised by persistently high levels of plasma aldosterone and inhibited renin secretion [4]. It has been considered that patients with PA have higher morbidity and mortality from cardiovascular or cerebrovascular diseases, compared with patients with primary hypertension [5]. Thus, given the high incidence of PA and the corresponding serious cardiovascular diseases it elicits, it is becoming essential for proper diagnosis and treatment of PA.

Specifically, on account of the variation in the management of different PA subtypes, screening PA and determining the dominant gland is of significant clinical value before medical treatment. For instance, aldosterone-producing adenomas (APA) should be treated with adrenalectomy, while pharmacological therapy is appropriate for bilateral adrenal hyperplasia [6]. According to guidelines from the Japan Endocrine Society 2009, plasma renin activity (PRA), plasma aldosterone concentration (PAC), and PAC/PRA ratio (ARR) are used for screening PA in hypertensive patients [7]. For patients diagnosed with PA, it is recommended that abdominal computed tomography (CT) scan and AVS should be performed separately to verify the adrenal mass anatomically and functionally. AVS has been widely accepted as the "gold standard" for the subtyping of PA. However, AVS poses certain limitations in practical use due to its high failure rate in clinical practice, especially in conditions of failure in the right side [8-11]. Therefore, it is urgently needed to explore novel strategies to detect the dominant gland in PA patients.

In this study, our group retrospectively analysed the clinical outcomes of 116 patients who followed the management routine of PA in the West China Hospital of Sichuan University. We compared the aldosterone concentrations measured in the adrenal vein and peripheral blood to determine the dominant gland in 
patients when sampling failed in the right side. Our developed strategy might provide a better alternative for lateralisation diagnostics in PA patients.

\section{Material and methods}

\section{Study design and subjects}

In this retrospective analysis study, we followed up the clinical outcomes of patients who entered the clinical routine of PA management in the West China Hospital from April 2015 to April 2017. 133 patients diagnosed with PA in the endocrine department of West China hospital were sent for AVS and CT scan. Patients were enrolled in our study if they met the following criteria: 1 - AVS detected dominant gland with ipsilateral adrenal lesion(s) and no adrenal lesion found in the contralateral gland; 2 - patients with bilateral adrenal lesions treated with unilateral adrenalectomy if there was a dominant gland; and 3 - if there was no adrenal lesion found by $\mathrm{CT}$, adrenalectomy was performed when there was an AVS-detected dominant gland. Exclusion criteria included: 1 - drug addiction history $(\mathrm{n}=1) ; 2$ - contraindication for general anaesthesia $(\mathrm{n}=2)$; and 3 - no dominant gland found by AVS ( $\mathrm{n}$ $=14$ ). Collectively, a total of 116 cases of patients with PA were enrolled in our study. Their clinical features are listed in Table 1. The experimental design, methods of clinical data collection, and strategies of data evaluation were performed in accordance with the approved guidelines. Written, informed consent was obtained from each participant.

\section{Management routine for primary aldosteronism}

Primary aldosteronism screening is recommended to patients who have drug-resistant hypertension, unexplained spontaneous or diuretic-induced hypokalaemia, adrenal incidentaloma, sleep apnoea, a family history of early-onset hypertension, or a cerebrovascular accident at a young age ( $<40$ years). Patients with ARR values $>20(\mathrm{ng} / \mathrm{dL}) /(\mathrm{ng} / \mathrm{mL} \cdot \mathrm{h})$ and PAC values $>15 \mathrm{ng} / \mathrm{dL}$ should undergo captopril challenge test. Patients with PAC $>12$ $\mathrm{ng} / \mathrm{dL}$ after administration of $50 \mathrm{mg}$ captopril are diagnosed with PA and then transferred to the Urology Department for AVS. AVS and CT are used to establish a lateralisation diagnosis. Antihypertensives including angiotensin converting enzyme inhibitors (ACEI), angiotensin receptor blocker (ARB), $\beta$-receptor blocker, and dihydropyridine calcium antagonist must be stopped two weeks before AVS. Aldosterone receptor antagonist must be stopped at least four weeks before AVS. We choose an $\alpha$-receptor blocker to replace the interfering medications and to maintain blood pressure at its pre-medication-replacement level. The clinic features of all enrolled subjects, including blood pressure, blood biochemical tests, and drug dependence, were recorded before and after surgery. Laparoscopic total adrenalectomy was performed when a dominant gland was detected by AVS.

\section{Blood biochemical tests}

The plasma renin activity (PRA) was measured by radioimmunoassay using a commercial kit (Northern Institute of Biotechnology, Beijing, China). The plasma aldosterone concentration (PAC) was measured by radioimmunoassay using a commercial kit (Jiuding Biological Technology Ltd., Tian Jin, China).

\section{Adrenal vein sampling interpretation}

All AVS procedures were performed by an experienced interventional radiologist in a sequential fashion without cosyntropin stimulus. Before AVS was performed, the operator needs to identify the position of the adrenal gland and adrenal vein $(\mathrm{AV})$ from the contrast-enhanced CT. A 3-4 mL blood sample was obtained at each of the following four sites: inferior vena cava (IVC) below the confluence of the renal veins, IVC above the confluence of the
Table 1. Clinical features for all enrolled subjects

\begin{tabular}{|c|c|c|}
\hline \multicolumn{3}{|l|}{ Parameter } \\
\hline \multicolumn{3}{|l|}{ Sex } \\
\hline Male & 49 & (42.24\%) \\
\hline Female & 67 & $(57.76 \%)$ \\
\hline $\mathrm{BMI}\left[\mathrm{kg} / \mathrm{m}^{2}\right]$ & 24.21 & 3.25 \\
\hline Mean age [years] & 44.95 & 11.35 \\
\hline \multicolumn{3}{|l|}{ Blood pressure [mm Hg] } \\
\hline Systolic & 155.44 & 20.62 \\
\hline Diastolic & 98.59 & 15.92 \\
\hline \multicolumn{3}{|l|}{ Lying position } \\
\hline \multicolumn{3}{|l|}{ Plasma renin activity } \\
\hline Number of patients & 114 & \\
\hline Activity [ng/mL*h] & 0.1 & $0.04-0.21$ \\
\hline \multicolumn{3}{|l|}{ Plasma potassium } \\
\hline Number of patients & 114 & \\
\hline Concentration $[\mathrm{mmol} / \mathrm{L}]$ & 3.32 & 0.63 \\
\hline \multicolumn{3}{|l|}{ Plasma aldosterone } \\
\hline Number of patients & 114 & \\
\hline Concentration [ng/dL] & 32.11 & $25.13-39.05$ \\
\hline \multicolumn{3}{|l|}{ Plasma angiotensin 2} \\
\hline Number of patients & 114 & \\
\hline Concentration [ng/L] & 61.78 & 13.75 \\
\hline \multicolumn{3}{|l|}{ ARR } \\
\hline Number of patients & 97 & \\
\hline Value $[\mathrm{ng} / \mathrm{dL}] /\left[\mathrm{ng} / \mathrm{mL}^{*} \mathrm{~h}\right]$ & 309.27 & $139.24-765.08$ \\
\hline \multicolumn{3}{|l|}{ Standing position } \\
\hline \multicolumn{3}{|l|}{ Plasma renin activity } \\
\hline Number of patients & 113 & \\
\hline Activity [ng/mL*h] & 0.32 & $0.12-0.61$ \\
\hline \multicolumn{3}{|l|}{ Plasma potassium } \\
\hline Number of patients & 114 & \\
\hline Concentration [mmol/L] & 3.29 & 0.6 \\
\hline \multicolumn{3}{|l|}{ Plasma aldosterone } \\
\hline Number of patients & 113 & \\
\hline Concentration [ng/dL] & 29.42 & $23.81-35.55$ \\
\hline \multicolumn{3}{|l|}{ Plasma angiotensin 2} \\
\hline Number of patients & 113 & \\
\hline Concentration [ng/L] & 60.3 & $55.75-72.95$ \\
\hline \multicolumn{3}{|l|}{ ARR } \\
\hline Number of patients & 113 & \\
\hline Value $[\mathrm{ng} / \mathrm{dL}] /\left[\mathrm{ng} / \mathrm{mL}^{*} \mathrm{~h}\right]$ & 91.53 & $42.45-210.44$ \\
\hline \multicolumn{3}{|c|}{$\mathrm{BMI}$ — body mass index; ARR — aldosterone-to-renin ratio } \\
\hline \multicolumn{3}{|c|}{$\begin{array}{l}\text { All variables refer to baseline data unless otherwise stated. All quantitative } \\
\text { and normally distributed variables are reported as mean } \pm \text { standard deviation; } \\
\text { non-normally distributed variables are presented as median with lower and } \\
\text { upper quartiles in parentheses. P values }<0.05 \text { were considered statistically } \\
\text { significant }\end{array}$} \\
\hline
\end{tabular}


renal veins, left adrenal vein (LAV), and right adrenal vein (RAV). When taking blood samples from the RAV, the radiologist should firstly find a point of attachment for the catheter and then gently inject $3-5 \mathrm{ml}$ of iodine contrast medium into the catheter to confirm its position. If the appearance of contrast agent is observed in the adrenal gland under $\mathrm{X}$-ray, it confirms that the blood from the adrenal vein drains into the catheter.

When adrenal vein/inferior vena cava (IVC) cortisol ratios (selective index - SI) are $\geq 3: 1$, we considered this procedure as a successful cannulation. Lateralisation index (LI) was calculated by dividing the aldosterone to cortisol ratio of the dominant adrenal gland by that of the non-dominant gland. Unilateral disease is diagnosed if $L I \geq 2$ on the premise of successful cannulation. Patients with SI $<3$, which is considered as failed cannulation, were diagnosed using aldosterone index (AI) to determine the dominant gland. AI is calculated by dividing the adrenal vein aldosterone concentration by the aldosterone concentration of IVC. If the AI of one side is $<1$ and $>1$ on the contralateral side, we diagnosed the contralateral adrenal gland as the dominant gland. Otherwise, the patients were advised to accept drug therapy.

\section{Statistical analysis}

The statistical analysis was performed by an in-house script based on R software (https://www.r-project.org). The Kolmogorov-Smirnov test was used if the data showed a normal distribution. Also, if the data were normally distributed, Student's t-test was used to compare the difference of demographic data between different subgroups. Two-way repeated-measures ANOVA and Tukey's post hoc test were applied for the detection of significant differences between groups at different follow-up points. Accordingly, these values were presented as mean \pm standard error $(\mathrm{SD})$. When data were not normally distributed, the Wilcoxon signed-rank test was performed, and the values were listed as medians (upper-lower quartile). The drug dependence was tested with chi-square $\left(\chi^{2}\right)$ test, which was corrected by Fisher's exact test. Receiver operator characteristic (ROC) curve analysis was performed to calculate the predictive power of the sensitivity and specificity of our developed strategy. A two-tailed $\mathrm{p}$ value less than 0.05 was considered as significant, unless specifically indicated.

\section{Results}

\section{Demography of enrolled patients}

The clinical characteristics of all included patients are illustrated in Table I. The proportion of males was $42.24 \%$ and females $57.76 \%$. The age, body mass index (BMI), and blood pressure (systolic blood pressure/diastolic blood pressure) for all the patients was $44.95 \pm 11.35$ years, $24.21 \pm 3.25 \mathrm{~kg} / \mathrm{m}^{2}$, and $155.44 \pm 20.62 / 98.59 \pm 15.92 \mathrm{~mm} \mathrm{Hg}$, respectively. For primary screening of PA in hypertensive patients, we measured the plasma potassium levels, PRA, angiotensin 2 concentrations, and PAC, as well as ARR, for individuals in lying and standing positions. The mean concentrations of these biochemical parameters in the lying position were $3.32 \pm 0.63 \mathrm{mmol} / \mathrm{L}, 0.1(0.04-0.21)$ $\mathrm{ng} / \mathrm{mL} \cdot \mathrm{h}, 61.78 \pm 13.75 \mathrm{ng} / \mathrm{L}, 32.11(25.13-39.05)$ $\mathrm{ng} / \mathrm{dL}$, and 309.27 (139.24-765.08) (ng/dL)/(ng/mL-h), respectively. In the standing position, these values were $3.29 \pm 0.6 \mathrm{mmol} / \mathrm{L}, 0.32(0.12-0.61) \mathrm{ng} / \mathrm{mL} \cdot \mathrm{h}, 60.3$ (55.75-72.95) ng/L, $29.42(23.81-35.55) \mathrm{ng} / \mathrm{dL}$, and 91.53 (2.45-210.44) (ng/dL)/(ng/mL·h), respectively.

\section{The aldosterone in AVS/peripheral blood ratio could be used to determine the dominant gland}

As illustrated in Figure 1, all patients experienced decreased SBP, DBP, and aldosterone and increased renin activity after treatment, which implied a recovery of PA. As mentioned above, we conducted a modified method to identify the dominant gland. Patients with dominant adrenal gland based on successful AVS were included in subgroup $2(n=75)$, while the patients who would undergo the operation based on the existence of a suppressed side after a failed sampling in the right side were enrolled in subgroup $1(n=41)$. The clinical features of the two groups are listed in Supplementary file: Table S1 and Table S2, separately. The clinical outcomes of patients in subgroup 1 and 2 were compared and are exhibited in Figure 1. The systolic and diastolic blood pressures were not significantly different before surgery in these two subgroups (Fig. 1, p value $>0.05$ ). The most influential indicators of PRA, ARR, and PAC were similar in subgroup 1 and 2 before surgery (Fig. 1, $\mathrm{p}$ value $>0.05)$, which indicated that the baseline conditions in these two groups were similar before surgery. In addition, there were no significant differences of blood pressure between subgroup 1 and 2 in the follow-up periods ( $\mathrm{p}$ value for sixth month $>0.05$, $p$ value for the $12^{\text {th }}$ month $>0.05$ ). The plasma angiotensin and aldosterone in subgroup 1 and 2 were not statistically significant for the sixth month ( $\mathrm{p}$ value for the sixth month $>0.05$ ) and were within normal range after 12 months. In brief, aldosterone, angiotensin, and blood pressure showed no obvious differences between the two subgroups after operation and were within the normal range after 12 months. Exhibiting similar clinical results from our developed method to those recommended in guidelines, it can be concluded that the aldosterone concentration measured in AVS and peripheral blood was useful to determine the dominant gland in patients with failed sampling in the right side. Moreover, the drug dependence in patients in subgroup 1 decreased significantly before and after surgery (Tab. 2 , p value $<0.05$ ). The patients who took at least one class of drug decreased from 40 to 10 after surgery. The drug dependence in patients in subgroup 2 decreased remarkably before and after surgery (Tab. 3, $\mathrm{p}$ value $<0.05$ ). The patients who take at least one class of drug decreased from 75 to 13 after surgery. As illustrated in Figure 2, AUC $=0.76$, sensitivity - 91.3\%, specificity $-67.53 \%$, likelihood ratio -2.81 , ROC analysis was performed to test the specificity and sensitivity based on AI in subgroup 2. The AUC for dominant gland detection was 0.76 , which resulted in $91.3 \%$ sensitivity and $67.53 \%$ specificity. The positive and negative likelihood ratios were 2.81 . 


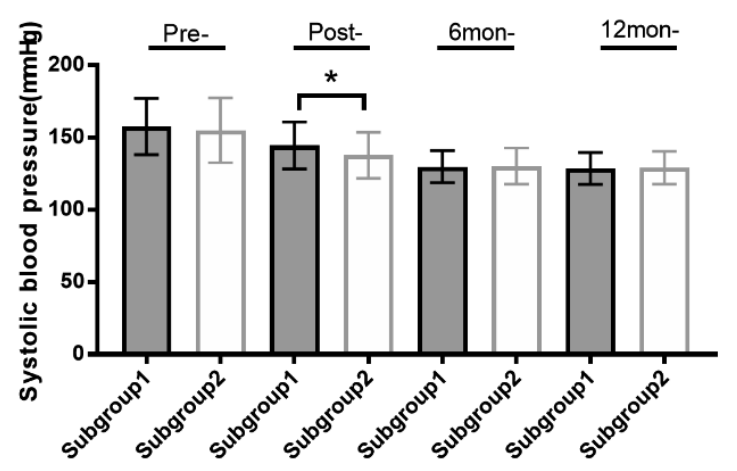

(A)

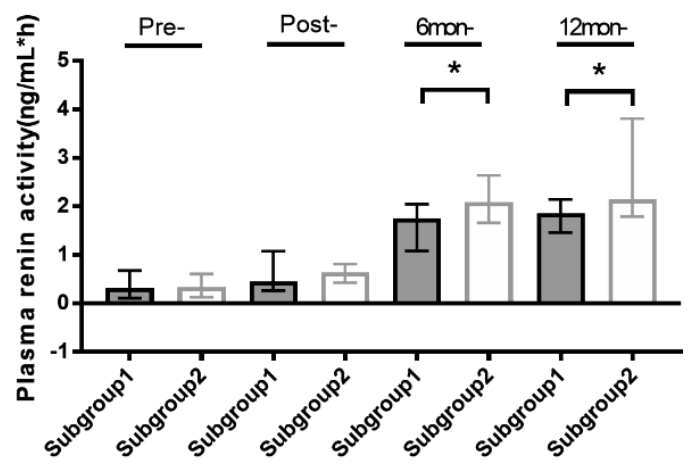

(C)

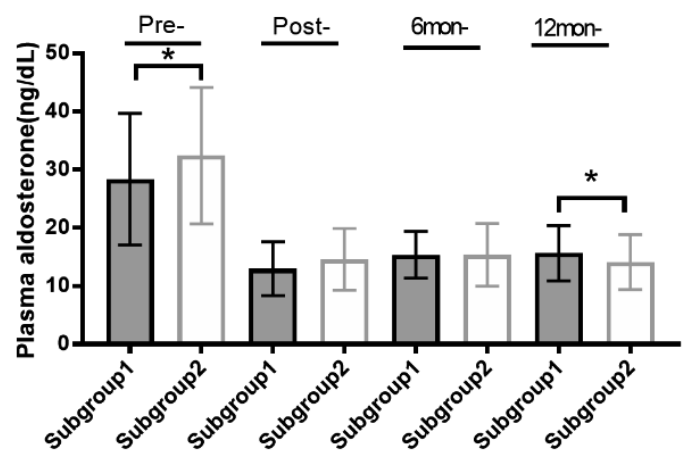

(E)

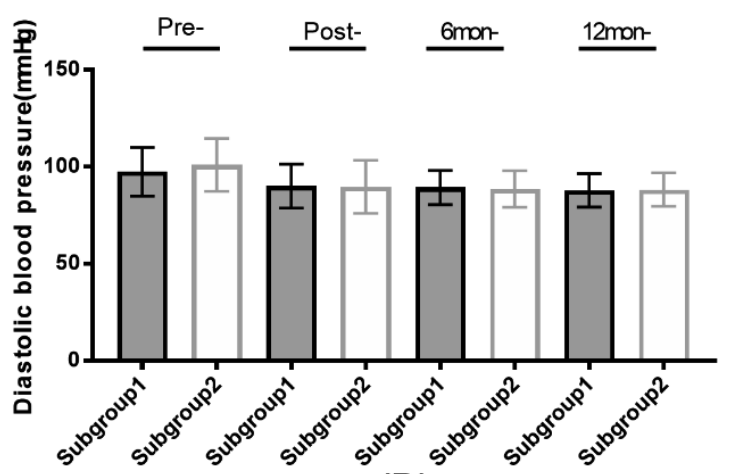

(B)

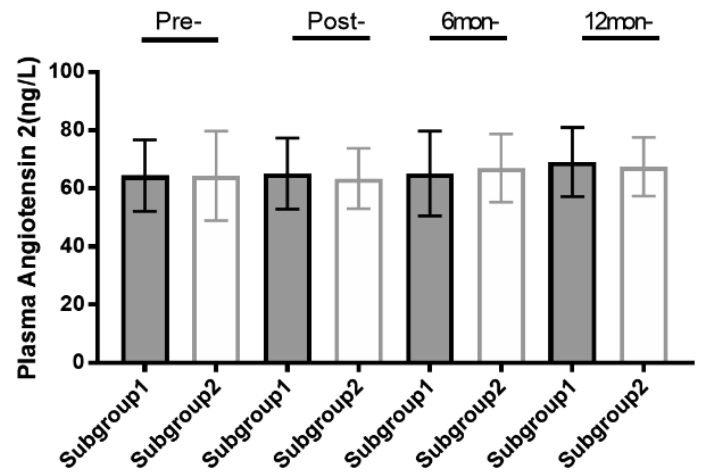

(D)

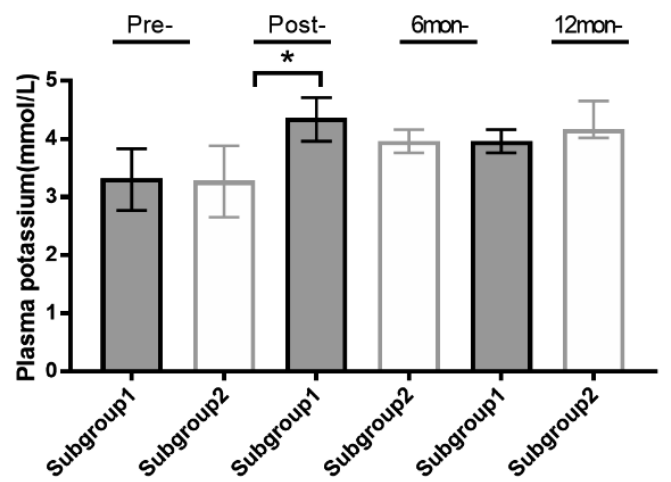

( $F)$

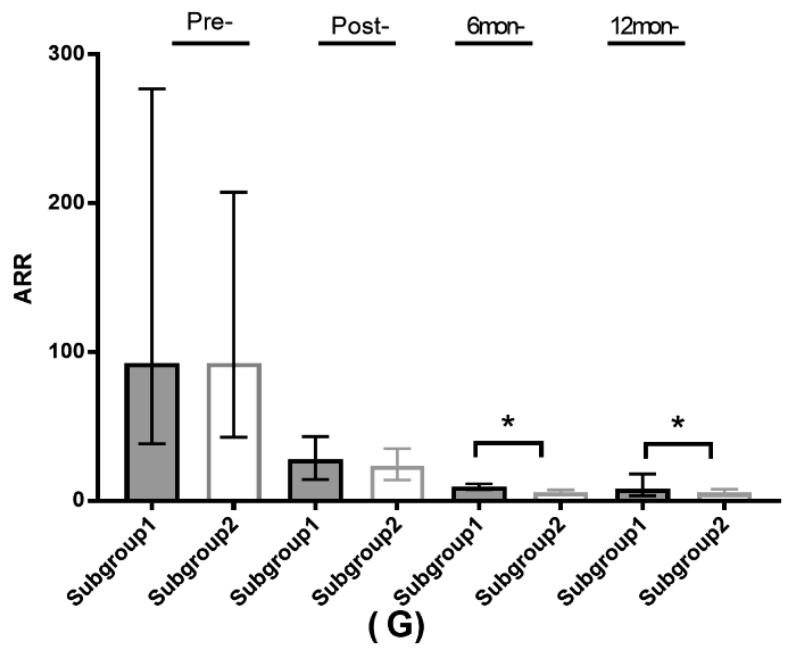

Figure 1A-G. Comparison of outcomes between subgroups 1 and 2 
Table 2. Comparison of drug dependence in patients in subgroup 1 before and after surgery

\begin{tabular}{lcccc}
\hline Classes of drugs & Before surgery & $\mathbf{1}$ month after surgery & $\mathbf{6}$ months after surgery & p value \\
\hline 0 & 1 & 31 & 31 & $0.00^{*}$ \\
\hline 1 & 22 & 9 & 9 & 1 \\
\hline 2 & 13 & 1 & 0 & \\
\hline 3 & 5 & 0 & & \\
\hline
\end{tabular}

$\mathrm{n}=41 ;{ }^{*} \mathrm{p}<0.05$, calculated by $\chi^{2}$ test

Table 3. Comparison of drug dependence in patients in subgroup 2 before and after surgery

\begin{tabular}{lcccc}
\hline Classes of drugs & Before surgery & $\mathbf{1}$ month after surgery & $\mathbf{6}$ months after surgery & p value \\
\hline 0 & 0 & 62 & 58 & $0.00^{*}$ \\
\hline 1 & 35 & 11 & 15 & \\
\hline 2 & 33 & 2 & 0 & \\
\hline 3 & 7 & 0 & 2 & \\
\hline
\end{tabular}

$\mathrm{n}=75 ;{ }^{*} \mathrm{p}<0.05$, calculated by $\chi^{2}$ test

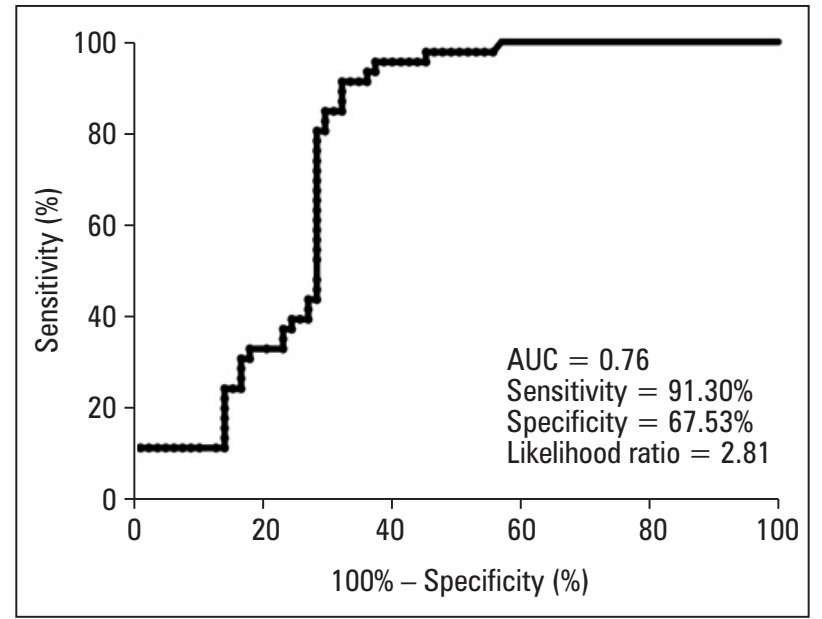

Figure 2. Receiver operator characteristic (ROC) curve analysis results. AUC - area under the curve

\section{Patients' age and gender showed close correlation to clinical outcome}

For patients with failed sampling in the right side and using AI to determine the dominant gland, we wondered if the factors of age, gender, and tumour location could affect the clinical outcomes of these patients. The patients were sub-classified according to age ( $<60$ or $>60$ years old; listed in Fig. 3), gender (male or female; listed in Fig. 4), and tumour location (left or right; listed in supplemental Fig. S1).

Interestingly, the SBP for patients elder than 60 years was higher than for those younger than 60 years after six months and 12 months (Fig. 3, p in sixth month $<0.05$, $p$ in $12^{\text {th }}$ month $\left.<0.05\right)$. DBP showed an identical variation trend as SBP (Fig. 3, p in $12^{\text {th }}$ month $<0.05$ ). In addition, the SBP for male patients was also higher than female patients after six months and 12 months (Fig. 4, $\mathrm{p}$ in sixth month $<0.05, \mathrm{p}$ in $12^{\text {th }}$ month $\left.<0.05\right)$. No significant differences of these indicators between left and right tumour were detected (supplemental Fig. 1, all $p>0.05$ ). These data suggested that the strategy for determination of dominant gland in our routine might be affected by age and gender.

\section{Discussion}

In this retrospective study, we developed a modified strategy for detecting the dominant gland in primary aldosteronism, and compared their clinical results with patients who underwent a successful cannulation in the right adrenal vein. For modified strategy in subtyping PA, the aldosterone levels in the adrenal vein and IVC were employed for the determination of the dominant gland in patients when sampling in the right side had failed. No significant differences in clinical outcome between this way and successful AVS were identified.

Primary aldosteronism is one of the most common causes of endocrine hypertension [12]. The pathogenesis of PA is still unclear at present. Generally, patients with PA exhibited a state of high plasma aldosterone levels and low plasma renin concentrations, which were caused by continuously excessive autonomous synthesis and release of aldosterone. Multiple pathologies of PA have been reported. About two-thirds of PA patients were attributed to bilateral adrenal hyperplasia, while aldosterone-producing adenomas accounted for about one-third of the patients with excessive aldosterone and insufficient renin activity [13]. The overgeneration of aldosterone in PA was reported to show various detrimental effects, such as cardiovascular events including 


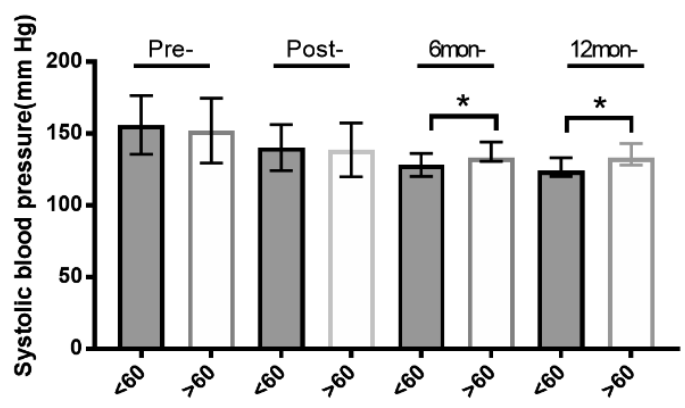

(A)

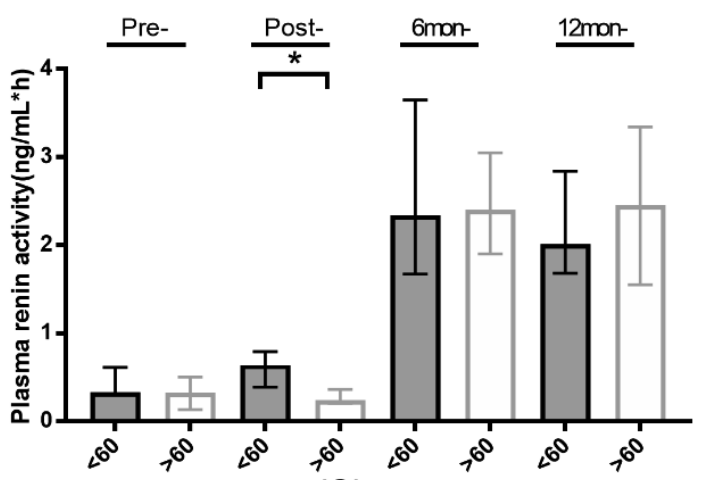

(C)

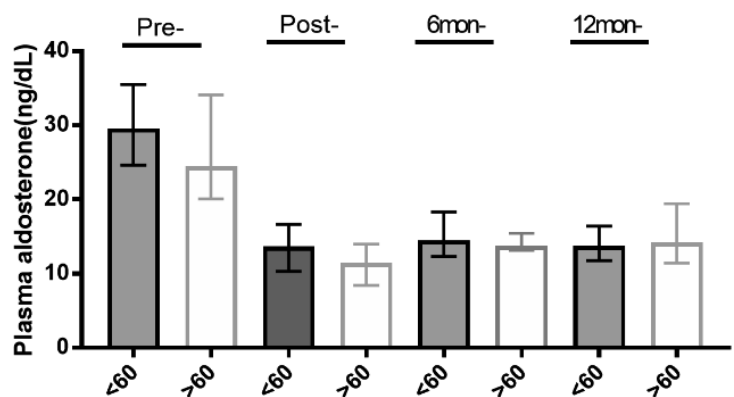

(E)

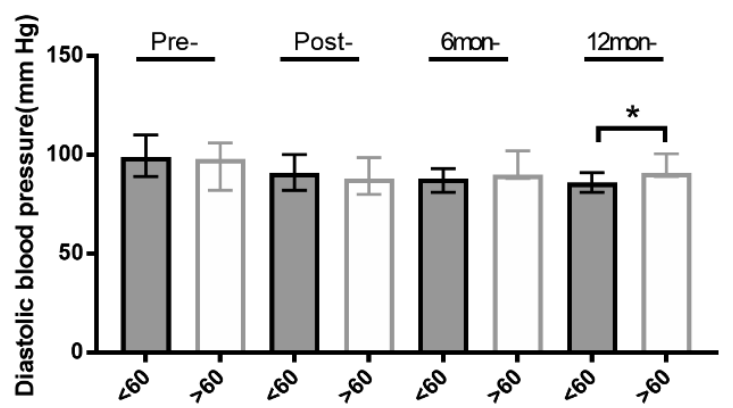

(B)

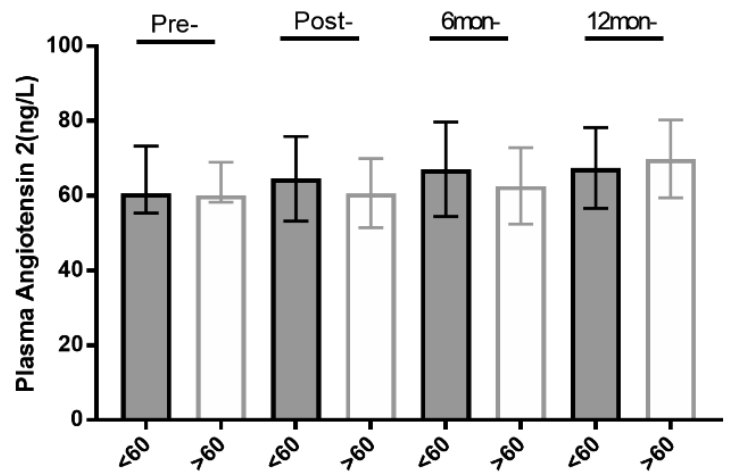

(D)

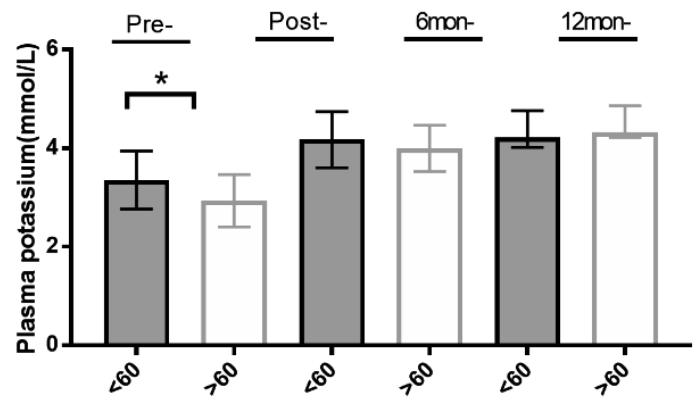

(F)

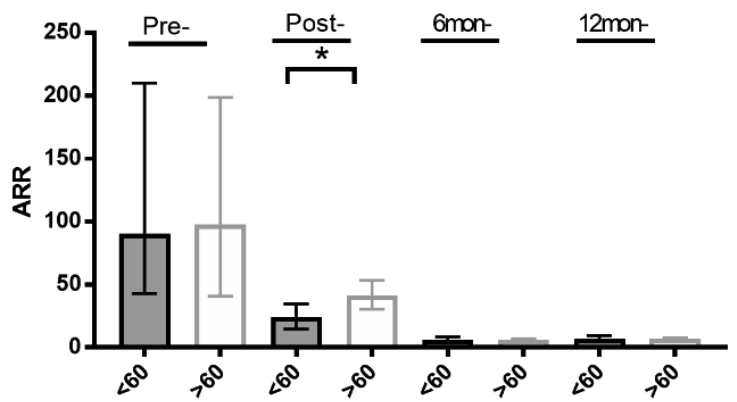

(G)

Figure 3A-G. Clinical features for all enrolled subjects who are younger than 60 years and older than 60 years

cerebrovascular accidents and myocardial infarctions, uncontrolled blood pressure, disorders of function and metabolism, etc. [14-20].

Due to the widespread prevalence in hypertensive patients [21] and poor outcomes of these patients [22], it is of great importance for early screening and diagnosis of PA. Currently, the recommended tests for screening the PA include measurement of plasma PRA, PAC, and ARR [23]. In this study, we screened the cases of high aldosterone and low renin activity in hypertensive patients. Captopril-challenge test or saline-loading test were also performed in this study, to establish the diag- 


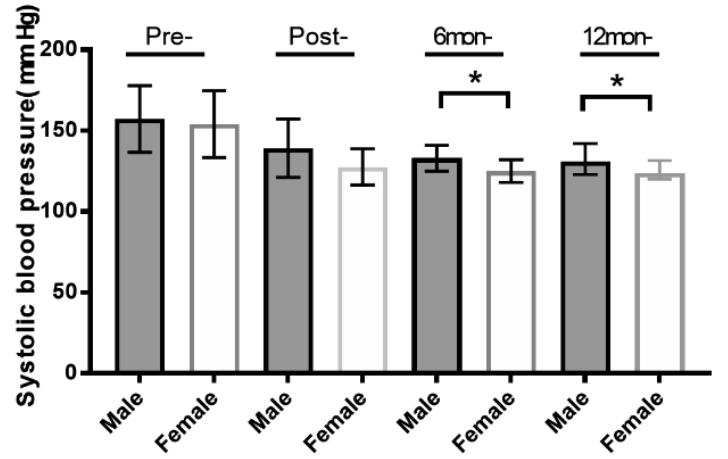

(A)

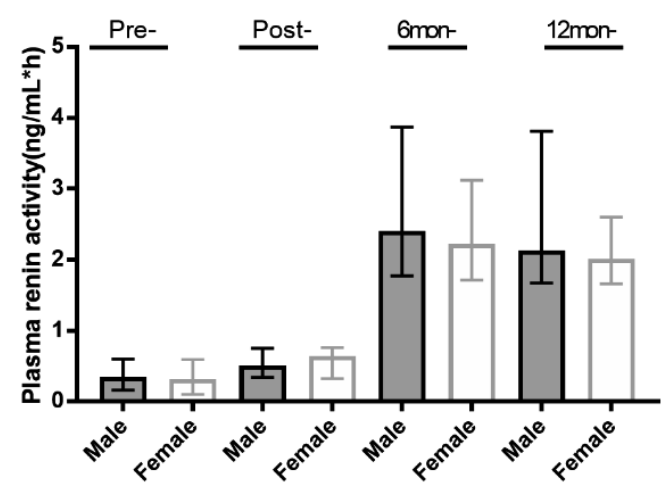

(C)

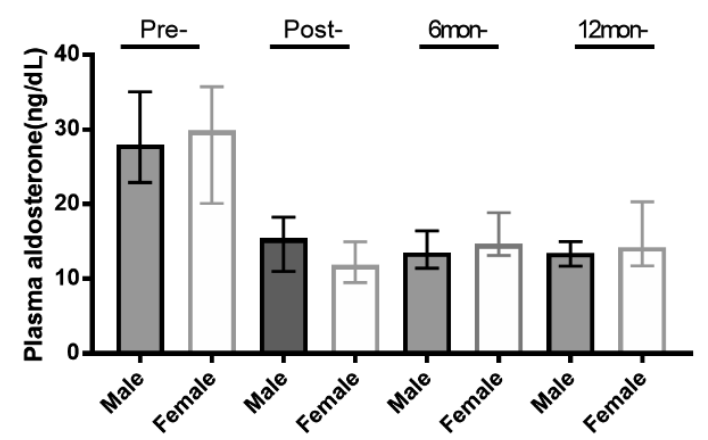

(E)

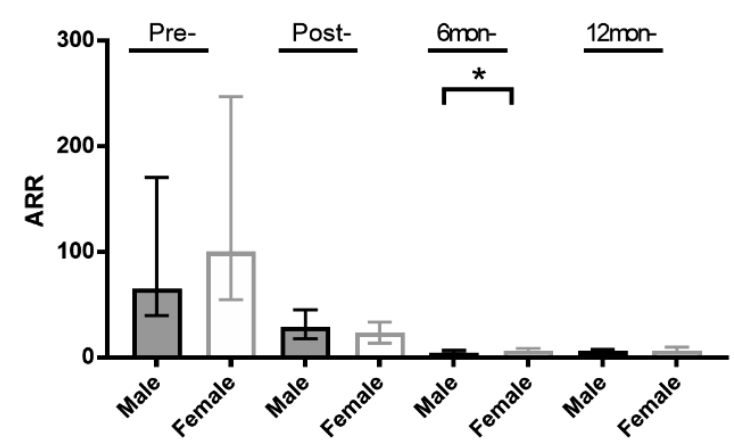

(G)

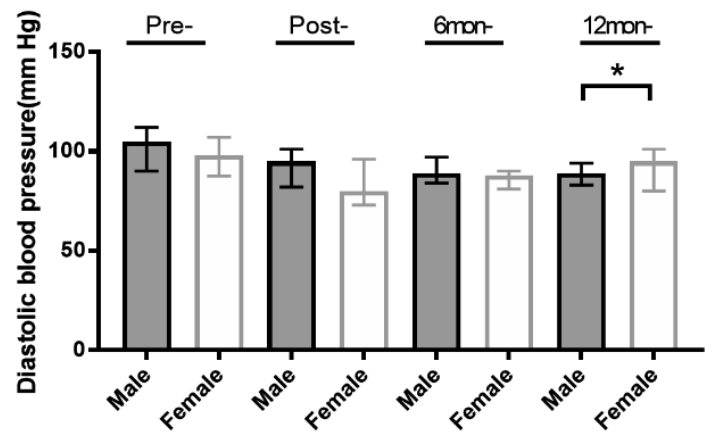

(B)

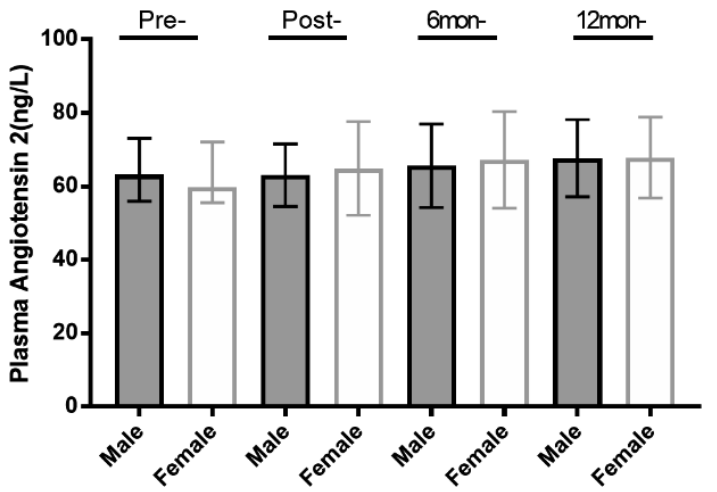

(D)

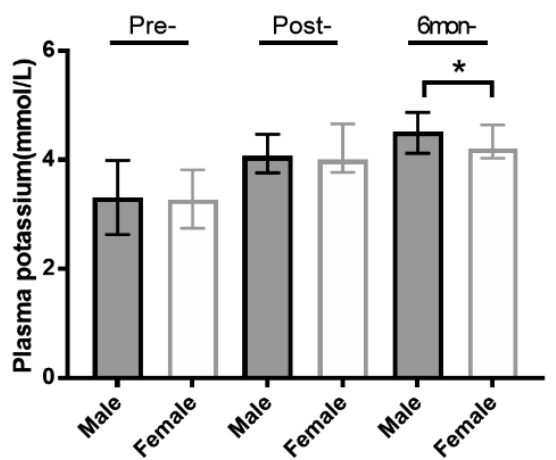

(F)

Figure 4A-G. Clinical features for all enrolled male subjects and female patients 
nosis of PA. The patients who met the diagnosed criteria of PA were sent for $\mathrm{CT}$ and AVS as routine management. The specificity and sensitivity of CT for PA subtyping was about $71 \%$ and $87 \%$, respectively [24]. The CT scan is optimal for detection of anatomical lesions in the adrenal gland, but it fails to identify the functional abnormalities. A retrospective study showed that about $37.8 \%$ of the patients with CT diagnosis of PA subtype were inconsistent with the results from AVS [22]. Approximately $14.6 \%$ of cases with AVS-identified bilateral aldosterone over-secretion showed unilateral lesions in CT scanning; about $19.1 \%$ of patients with AVS confirmed with unilateral aldosterone over-secretion were demonstrated to suffer bilateral lesions in CT scanning. In $3.9 \%$ patients, adrenalectomy was performed on the wrong side, which means in those patients the opposite adrenal gland was the AVS confirmed dominant gland. Although a recent study revealed no significant difference of PA diagnosis and clinical benefits between CT and AVS, the AVS was generally considered as the gold standard for PA subtyping. We combined CT and AVS in this study for PA subtyping.

Adrenal vein sampling is not easy to achieve, due to the technique and varied anatomical structure of the adrenal vein, especially when obtaining a blood sample from the right side. The anatomy for confluence of the right adrenal vein and inferior vena cava (IVC) is complex. In addition, the angle of these two veins is inconvenient for intubation, which always leads to failure of sampling. For patients with failed sampling in the right side there are no consistent recommendations for determination of the dominant gland. In practical use, right adrenal venous sampling often contains a mixture of adrenal venous blood and peripheral blood because the blood sample from the right adrenal vein is usually obtained at the peristome of the confluence where the right adrenal vein is collected into the IVC. In some cases, the RAV forms a common trunk with an accessory right hepatic vein before it drains into the IVC, which makes it almost impossible to obtain an RAV blood sample without the mixture of blood from the right hepatic vein. As a result, the SI value could be lower than 3 and the sampling could be defined as a failure, which might mislead the subsequent therapeutic choice. In this study, we, for the first time, used the aldosterone concentrations to directly determine the dominant gland. The pathophysiological basis for this strategy is that the aldosterone concentration in the right adrenal venous blood or even in mixture of adrenal venous blood and peripheral blood should be higher than that in the peripheral blood in normal conditions. Only when the high concentration of aldosterone in one side, caused by high autonomous aldosterone secretion from APA, inhibits the activity of renin angiotensin aldosterone system (RAAS), will the aldosterone secretion of the contralateral adrenal gland be suppressed; as a result, the aldosterone concentration of the contralateral side could be lower than that in the peripheral blood. According to the clinical outcomes in these patients, determination of the dominant gland by this strategy is effective in clinical practice. A larger clinical study containing a larger sample size is now being conducted by our group to verify these findings.

Our study results provided evidence of using aldosterone index to diagnose the dominant gland when AVS falied on one side. Though as illustrated in the AUC curve, the specificity is not high, it could be an alternative method especially currently there is lack of effective method to make a lateralization diagnosis when sampling failed on one side.

\section{Competing interests}

All authors reviewed and approved the manuscript. The authors have declared that there exist no competing interests.

\section{Authors' contributions}

YC.Z conceived and/or designed the work; T.C., Z.H.L. acquired data; ZJ.R., S.Z.L. prepared the figures; and SZ.L. and L.Z. wrote the paper. S.Z.L and L.Z. contributed equally to this work.

\section{References}

1. Hannemann A, Wallaschofski H. Prevalence of primary aldosteronism in patient's cohorts and in population-based studies--a review of the current literature. Horm Metab Res. 2012; 44(3): 157-162, doi: 10.1055/s-0031-1295438, indexed in Pubmed: 22135219.

2. Omura M, Saito J, Yamaguchi K, et al. Prospective study on the prevalence of secondary hypertension among hypertensive patients visiting a general outpatient clinic in Japan. Hypertens Res. 2004; 27(3): 193-202, indexed in Pubmed: 15080378

3. Douma S, Petidis K, Doumas M, et al. Prevalence of primary hyperaldosteronism in resistant hypertension: a retrospective observational study. Lancet. 2008; 371(9628): 1921-1926, doi: 10.1016/S0140-6736(08)60834-X, indexed in Pubmed: 18539224

4. Conn JW. Primary hyperaldosteronism. A new clinical syndrome. J Lab Clin Med. 1955; 45(1): 3-14, indexed in Pubmed: 13233623

5. Mulatero P, Monticone S, Bertello C, et al. Long-term cardio- and cerebrovascular events in patients with primary aldosteronism. J Clin Endocrinol Metab. 2013; 98(12): 4826-4833, doi: 10.1210/jc.2013-2805, indexed in Pubmed: 24057288.

6. Mulatero P, Dluhy RG, Giacchetti G, et al. Diagnosis of primary aldosteronism: from screening to subtype differentiation. Trends Endocrinol Metab. 2005; 16(3): 114-119, doi: 10.1016/j.tem.2005.02.007, indexed in Pubmed: 15808809

7. Nishikawa T, Omura M, Satoh F, et al. Task Force Committee on Primary Aldosteronism, The Japan Endocrine Society. Guidelines for the diagnosis and treatment of primary aldosteronism - the Japan Endocrine Society 2009. Endocr J. 2011; 58(9): 711-721, doi: 10.1507/endocrj. ej11-0133, indexed in Pubmed: 21828936.

8. Espiner EA, Ross DG, Yandle TG, et al. Predicting surgically remedial primary aldosteronism: role of adrenal scanning, posture testing, and adrenal vein sampling. J Clin Endocrinol Metab. 2003; 88(8): 3637-3644, doi: 10.1210/jc.2002-022051, indexed in Pubmed: 12915648.

9. Monticone S, Viola A, Rossato D, et al. Adrenal vein sampling in primary aldosteronism: towards a standardised protocol. Lancet Diabetes Endocrinol. 2015; 3(4): 296-303, doi: 10.1016/S2213-8587(14)70069-5, indexed in Pubmed: 24831990. 
10. Funder JW, Carey RM, Fardella C, et al. Endocrine Society. Case detection, diagnosis, and treatment of patients with primary aldosteronism: an endocrine society clinical practice guideline. J Clin Endocrinol Metab. 2008; 93(9): 3266-3281, doi: 10.1210/jc.2008-0104, indexed in Pubmed: 18552288.

11. El Ghorayeb N, Mazzuco TL, Bourdeau I, et al. Basal and Post-ACTH Aldosterone and Its Ratios Are Useful During Adrenal Vein Sampling in Primary Aldosteronism. J Clin Endocrinol Metab. 2016; 101(4): 1826-1835, doi: 10.1210/jc.2015-3915, indexed in Pubmed: 26918291.

12. Rossi GP, Bernini G, Caliumi C, et al. PAPY Study Investigators. A prospective study of the prevalence of primary aldosteronism in 1,125 hypertensive patients. J Am Coll Cardiol. 2006; 48(11): 2293-2300, doi: 10.1016/j.jacc.2006.07.059, indexed in Pubmed: 17161262.

13. Matsumura K, Fujii K, Oniki H, et al. Role of aldosterone in left ventricular hypertrophy in hypertension. Am J Hypertens. 2006; 19(1): 13-18, doi: 10.1016/j.amjhyper.2005.05.013, indexed in Pubmed: 16461184.

14. Novello M, Catena C, Nadalini E, et al. Renal cysts and hypokalemia in primary aldosteronism: results of long-term follow-up after treatment. J Hypertens. 2007; 25(7): 1443-1450, doi: 10.1097/HJH.0b013e328126855b, indexed in Pubmed: 17563567.

15. Rossi GP, Belfiore A, Bernini G, et al. Primary Aldosteronism Prevalence in hYpertension Study Investigators. Body mass index predicts plasma aldosterone concentrations in overweight-obese primary hypertensive patients. J Clin Endocrinol Metab. 2008; 93(7): 2566-2571, doi: 10.1210/jc.2008-0251, indexed in Pubmed: 18445663.

16. Krug AW, Ehrhart-Bornstein M. Aldosterone and metabolic syndrome: is increased aldosterone in metabolic syndrome patients an additional risk factor? Hypertension. 2008; 51(5): 1252-1258, doi: 10.1161/HYPERTENSIONAHA.107.109439, indexed in Pubmed: 18347227.

17. Fujita T. Aldosterone and CKD in metabolic syndrome. Curr Hyperten Rep. 2008; 10(6): 421-423, doi: 10.1007/s11906-008-0078-y.
18. Sim JJ, Yan EH, Liu InL, et al. Positive relationship of sleep apnea to hyperaldosteronism in an ethnically diverse population. J Hypertens. 2011; 29(8): 1553-1559, doi: 10.1097/HJH.0b013e3283492219, indexed in Pubmed: 21720263.

19. Salcuni AS, Palmieri S, Carnevale V, et al. Bone involvement in aldosteronism. J Bone Miner Res. 2012; 27(10): 2217-2222, doi: 10.1002/jbmr.1660, indexed in Pubmed: 22589146.

20. Williams TA, Lenders JWM, Mulatero P, et al. Primary Aldosteronism Surgery Outcome (PASO) investigators. Outcomes after adrenalectomy for unilateral primary aldosteronism: an international consensus on outcome measures and analysis of remission rates in an international cohort. Lancet Diabetes Endocrinol. 2017; 5(9): 689-699, doi: 10.1016/S2213-8587(17)30135-3, indexed in Pubmed: 28576687.

21. Mulatero P, Bertello C, Rossato D, et al. Roles of clinical criteria, computed tomography scan, and adrenal vein sampling in differential diagnosis of primary aldosteronism subtypes. J Clin Endocrinol Metab. 2008; 93(4): 1366-1371, doi: 10.1210/jc.2007-2055, indexed in Pubmed: 18198224

22. Kempers MJE, Lenders JWM, van Outheusden L, et al. Systematic review: diagnostic procedures to differentiate unilateral from bilateral adrenal abnormality in primary aldosteronism. Ann Intern Med. 2009; 151(5): 329-337, doi: 10.7326/0003-4819-151-5-200909010-00007, indexed in Pubmed: 19721021.

23. Dekkers T, Prejbisz A, Kool LJ, et al. SPARTACUS Investigators. Adrenal vein sampling versus $C T$ scan to determine treatment in primary aldosteronism: an outcome-based randomised diagnostic trial. Lancet Diabetes Endocrinol. 2016; 4(9): 739-746, doi: 10.1016/S2213-8587(16)30100-0, indexed in Pubmed: 27325147.

24. Johnstone FR. The suprarenal veins. Am J Surg. 1957; 94(4): 615-620, doi: 10.1016/0002-9610(57)90590-1, indexed in Pubmed: 13458646. 